\title{
The distinct mechanisms of the antitumor activity of emodin in different types of cancer (Review)
}

\author{
WEI-TIAN WEI ${ }^{1}$, SHENG-ZHANG LIN ${ }^{2}$, DIAN-LEI LIU ${ }^{3}$ and ZHAO-HONG WANG ${ }^{4}$ \\ ${ }^{1}$ Department of Oncological Surgery, Zhejiang Cancer Hospital; ${ }^{2}$ Department of Hepato-biliary-pancreatic Surgery, \\ First Affiliated Hospital, Zhejiang University School of Medicine; ${ }^{3}$ Department of Surgery, Hangzhou Hospital \\ of Traditional Chinese Medicine, Hangzhou 310005; ${ }^{4}$ Department of Hepato-biliary-pancreatic Surgery, \\ The Second Affiliated Hospital of Wenzhou Medical College, Wenzhou 325027, P.R. China
}

Received June 21, 2013; Accepted August 6, 2013

DOI: $10.3892 / o r .2013 .2741$

\begin{abstract}
Emodin, a tyrosine kinase inhibitor, is a natural anthraquinone derivative found in the roots and rhizomes of numerous plants. The inhibitory effect of emodin on mammalian cell cycle modulation in specific oncogene-overexpressing cells has formed the basis for using this compound as an anticancer drug. Previous reviews have summarized the antitumor properties of emodin. However, the specific molecular mechanisms of emodin-mediated tumor inhibition have not been completely elucidated over the last 5 years. Recently, there has been great progress in the preclinical study of the anticancer mechanisms of emodin. Our recent study revealed that emodin has therapeutic effects on pancreatic cancer through various antitumor mechanisms. Notably, the therapeutic efficacy of emodin in combination with chemotherapy was found to be higher than the comparable single chemotherapeutic regime, and the combination therapy also exhibited fewer side-effects. Despite these encouraging results, further investigation is warranted as emodin has been shown to modulate one or more key regulators of cancer growth. This review provides an overview of the distinct mechanisms of anticancer action of emodin in different body systems identified over the past 5 years. These new breakthrough findings may have important implications for targeted cancer therapy and for the future clinical use of emodin.
\end{abstract}

\section{Contents}

1. Introduction

2. Effects of emodin on digestive system cancer and the possible mechanisms involved

Correspondence to: Professor Sheng-Zhang Lin, Department of Hepato-biliary-pancreatic Surgery, First Affiliated Hospital, Zhejiang University School of Medicine, Hangzhou 310005, P.R. China

E-mail:wzf21sz@163.com

Key words: emodin, cancer, mechanism
3. Effects of emodin on respiratory system cancer and the possible mechanisms involved

4. Effects of emodin on reproductive system cancer and the possible mechanisms involved

5. Effects of emodin on blood system cancer and the possible mechanisms involved

6. Conclusions

\section{Introduction}

As a tyrosine kinase inhibitor, emodin (1,3,8-trihydroxy6-methyl-anthraquinone) (Fig. 1) is a natural anthraquinone derivative found in the roots and rhizomes of numerous plants. Pharmacological studies have demonstrated that emodin exhibits various biological functions, such as anti-inflammatory, anti-bacterial and anticancer activity. Studies have demonstrated that emodin inhibits cell growth in several types of cancer cells (1-3) and regulates genes related to the control of cell apoptosis, oncogenesis, cell proliferation, cancer cell invasion and metastasis (4-8). Notably, previous studies have identified many types of drugs that strengthen the therapeutic effect of gemcitabine on pancreatic cancer (9-12). Similarly, our recent study also concluded that emodin enhances the therapeutic effect of gemcitabine in pancreatic cancer without additional toxic effects (13). Although many researchers have reported the antitumor effects of emodin in several types of tumor cells, its specific molecular anticancer mechanisms have not been fully elucidated. A recent resurgence of research into the anticancer effects of emodin over the past 5 years has shown great progress. Emodin was found to have antitumor effects in different types of cancer, however, via different mechanisms. Therefore, the exact antitumor mechanisms of emodin require further study. The present review discusses the pharmacological activities of emodin and the mechanisms that induce cell death in many types of human cancer cells, both in vitro and in vivo. Research findings on emodininduced cytotoxicity and its protective effects against cancer in different body systems as well as the antitumor mechanisms involved are described below. 


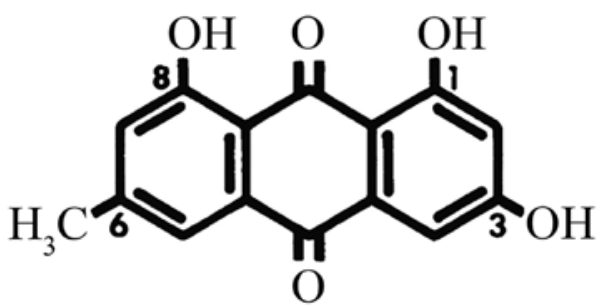

Figure 1. Structure of emodin, 1,3,8-trihydroxy-6-methyl-anthraquinone.

\section{Effects of emodin on digestive system cancer and the possible mechanisms involved}

Human tongue squamous cancer. Lin et al (14) reported that emodin mediated oxidative injury (DNA damage) based on reactive oxygen species (ROS) production and endoplasmic reticulum (ER) stress based on the levels of GADD153 and GRP78 that act as an early and upstream change in the cell death cascade to caspase- and mitochondria-dependent signaling pathways, triggers mitochondrial dysfunction from Bcl-2 and Bax modulation, mitochondrial cytochrome $c$ release and caspase activation, consequently leading to apoptosis in SCC-4 cells. Chen et al (15) also reported that emodin induced DNA damage followed by the inhibition of expression of DNA repair-associated genes, including ataxia telangiectasia mutated (ATM), ataxia-telangiectasia and Rad3-

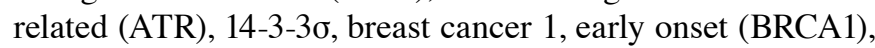
DNA-dependent serine/threonine protein kinase (DNA-PK) and $\mathrm{O}^{6}$-methylguanine-DNA methyltransferase (MGMT) in SCC-4 human tongue cancer cells. Moreover, they found that emodin inhibited the migration and invasion of SCC-4 cells and inhibited the protein levels and activity of matrix metalloproteinase-2 (MMP-2), without affecting gene expression of MMP-2, however, it inhibited the gene expression of MMP-9. MMP-9 (gelatinase-B) is known to play an important role and is associated with tumor migration, invasion and metastasis in various types of human cancers. Furthermore, the authors demonstrated that emodin decreased the levels of MMP-2 and urokinase plasminogen activator (u-PA) in a concentrationdependent manner (16).

Gastric cancer and esophageal cancer. RhoA expression was found to be upregulated in primary gastric carcinoma compared with normal gastric mucosa as reported by Cai et al (17). Emodin-induced generation of ROS was found to inhibit RhoA activation to sensitize gastric carcinoma cells to anoikis (17). Phosphatase of regenerating liver-3 (PRL3), a novel gene, has been found to play an important role in the promotion of cellular proliferation as well as inhibition of apoptosis in cancer cells. Sun and $\mathrm{Bu}(18)$ revealed that emodin inhibited cell growth and induced apoptotic cell death in the SGC-7901 human gastric carcinoma cell line via downregulation of PRL-3. Wang et al (19) reported that human esophageal carcinoma EC-109 cells treated with emodin underwent rapid apoptosis. Emodin led to the growth inhibition of EC-109 cells in a time- and dose-dependent manner. Furthermore, the intracellular $\mathrm{pH}$ (pHi) was found to decrease significantly by 0.47-0.78 units after treatment with emodin. Bursts of ROS took place after the application of emodin (19). These results indicate that emodin may be a strong anticancer drug against esophageal cancer by causing various early events leading to tumor cell growth inhibition, including the production of ROS and a decrease in pHi, resulting in cellular apoptosis (19).

Liver cancer. Hsu et al (20) demonstrated that emodin caused G2/M arrest in several types of liver cancer cells (Huh7, Hep3B and HepG2) indicating that emodin has a broad spectrum of activity against human hepatoma cells. They revealed that 15 representative genes were associated with the emodin treatment response in hepatoma cell lines. These genes included CYP1A1, CYP1B1, GDF15, SERPINE1, SOS1, RASD1 and MRAS which were all upregulated, while NR1H4, PALMD and TXNIP were downregulated following treatment with emodin for 6 h. Finally, levels of the TIPARP, SLC7A11 and CYR61 genes were increased while IGFBP3 levels decreased at $24 \mathrm{~h}$ following emodin treatment. Yu et al (21) observed that emodin induced apoptosis in HepG2 cells and caused a significant accumulation of cells in the G1 phase. Emodin also caused an increase in cytochrome $c$ release into the cytosol from mitochondria and the activation of caspase- 9 and caspase- 8 . In addition, treatment with emodin resulted in a dose-dependent accumulation of intracellular ROS. Furthermore, the authors found that emodin increased the protein expression level of p53 and decreased the protein level of NF- $\mathrm{NB} / \mathrm{p} 65$ in HepG2 cells, which may play a role in emodin-induced apoptosis.

Gallbladder cancer. Gallbladder carcinoma is known to be resistant to many forms of anticancer drugs. For this reason, new cancer therapy strategies must be investigated for the future treatment and management of this disease. The major platinum-containing drugs including cisplatin, carboplatin and oxaliplatin are widely used anticancer agents for the treatment of various solid tumors, but have not been proven effective for treating gallbladder cancers (22). Wang et al (23) reported that co-treatment of cisplatin, carboplatin and oxaliplatin with emodin (an ROS generator) effectively enhanced the chemosensitivity of gallbladder carcinoma cell line SGC996 both in vitro and in vivo in comparison with cisplatin, carboplatin or oxaliplatin treatment alone. The mechanisms were attributed to emodin-induced reduction in the glutathione level, and downregulation of multidrug resistance-related protein 1 (MRP1) (a type of GS-X pump) expression in SGC996 cells. Remarkably, emodin exhibited few systemic toxic effects in vivo. This finding supports the notion that ROS manipulation strategy could be selective between cancerous and normal cells, as indicated by an increasing body of evidence $(24,25)$. The expression of survivin is involved in the inhibition of apoptosis. Wang et al (26) reported that emodin potentiated the antitumor effects of cisplatin (CDDP) on gallbladder cancer cells by generating ROS and by downregulating the expression of survivin without causing detectable toxic effects on normal tissues. Side population (SP) cells are likely responsible for tumor metastasis and recurrence since they exhibit enhanced potential for proliferation, clonogenicity, tumorigenicity and invasion (27-29). Li et al (30) isolated a small population of stem-like SP cells from the gallbladder carcinoma cell line, SGC-996. They found that SP cells displayed a higher proliferation rate, a higher clonal-generating capability, higher 
tumorigenic, migratory and invasive abilities than non-SP cells. Notably, they found that emodin reduced the ratio, inhibited colony formation and effectively eliminated sphere formation of SP cells. Furthermore, emodin sensitized SP cells to cisplatin to overcome chemo-resistance via inhibition of expression of ATP-binding cassette subfamily $\mathrm{G}$ member 2 (ABCG2). In addition, emodin/cisplatin co-treatment in vivo suppressed the tumor growth derived from SP cells through downregulation of ABCG2 expression (31).

Colon cancer. Lu et al (32) demonstrated that emodin inhibited cancer-cell growth by blocking vascular endothelial growth factor (VEGF) receptor signaling and revealed that emodin can be used as a potential inhibitor for colon cancer angiogenesis. Ma et al (33) reported that emodin induced apoptosis of LS1034 human colon cancer cells in vitro and inhibited tumor xenografts derived from LS1034 cells in vivo. During the in vitro study, emodin induced cell morphological changes, decreased the percentage of viability, induced G2/M phase arrest and increased $\mathrm{ROS}$ and $\mathrm{Ca}(2+)$ production as well as loss of mitochondrial membrane potential $(\Delta \Psi \mathrm{m})$ in LS1034 cells. Emodin-triggered apoptosis was also found to be concentration-dependent. The protein levels of caspase-9, cytochrome $c$ and the ratio of $\mathrm{Bax} / \mathrm{Bcl}-2$ were increased in LS1034 cells following emodin exposure. Their study suggests that emodin induces the production of $\mathrm{ROS}$ and $\mathrm{Ca}(2+)$ release and alters the levels of anti- and pro-apoptotic proteins, leading to mitochondrial dysfunction and activation of caspase- 9 and caspase-3 which in turn causes apoptosis in LS1034 cells. In the in vivo study, emodin effectively suppressed tumor growth in tumor xenografts bearing LS1034 cells but it was unknown whether emodin caused tumor inhibition via mitochondrial dysfunction as in vitro (33). Many colorectal cancer (CRC) patients suffer from the unexpected development of micrometastasis after curative resection of primary tumors, and $\sim 50 \%$ develop liver metastasis at some time point during their disease progression. PRL-3 is expressed at high levels in nearly all metastatic lesions that are derived from $\mathrm{CRC}$, regardless of the metastatic site. Yet weak or no PRL-3 expression is observed in normal colon and non-metastatic primary cancers (34). PRL-3 was found to promote metastasis in a diverse array of cancers (35). Han et al (36) found that emodin strongly inhibited phosphatase activity of PRL-3 in the colon cancer cell line DLD-1 and blocked PRL-3-induced tumor cell migration and invasion in a dose-dependent manner. Emodin rescued the phosphorylation of ezrin, which is a known PRL-3 substrate. The results of this study indicate that emodin is a PRL-3 inhibitor and a lead molecule for development of a selective PRL-3 inhibitor. MMP-2 and -9 (gelatinase A and B) are considered to be important biomolecules in colon cancer progression. Nitric oxide (NO), a free radical that plays an important role in signaling pathways, regulates MMP expression. In contrast, tumor cell-derived NO promotes the expression of angiogenic factors. Damodharan et al (37) demonstrated that emodin suppresses the NO-mediated upregulation of MMP-2 and -9. Thus, emodin can be selected as an effective antimetastatic agent in NO-induced tumor progression. Therefore, elucidation of critical pathways in metastasis, where emodin could exert its inhibitory effects, may make it 'a perfect fit' for antitumor therapy. Finally, emodin may be developed for the treatment of colon cancer in the future due to its potential ability to inhibit tumor growth and metastasis.

Pancreatic cancer. Survivin, a member of the inhibitor of apoptosis gene family, is involved in the control of cell division and inhibition of apoptosis and is described as a $\beta$-catenin/Tcf/Lef target gene. Guo et al (38) reported that emodin not only downregulated the expression of survivin and $\beta$-catenin but also decreased the translocation of $\beta$-catenin to the nucleus in pancreatic cancer and, thus, potentiated the antitumor effects of gemcitabine. A recent study by our research team suggests that emodin has a therapeutic effect on pancreatic cancer through various antitumor mechanisms (Fig. 2). In Chen et al (39), we demonstrated that tumors in nude mice subcutaneously injected with SW1990 cells and treated with a combination of emodin $(40 \mathrm{mg} / \mathrm{kg})$ and gemcitabine $(80 \mathrm{mg} / \mathrm{kg})$ showed a significant reduction in tumor volume, $\mathrm{Ki}-67$ proliferation index and expression of the $\mathrm{Bcl}-2 / \mathrm{Bax}$ ratio compared with tumors from mice treated with sodium chloride, emodin alone (40 mg/kg) or gemcitabine alone $(125 \mathrm{mg} / \mathrm{kg})$, and induced increased release of $\mathrm{CytC}$ from the mitochondria to the cytoplasm and triggered caspase- 3 activation leading to apoptosis. The results suggest that emodin improves the antitumor effect of gemcitabine, even at a lower dose of gemcitabine, so as to decrease the toxicity of chemotherapy on transplanted tumors derived from the SW1990 cell line via the enhancement of apoptosis induced by gemcitabine. This mechanism may involve the downregulation of the $\mathrm{Bcl}-2 / \mathrm{Bax}$ ratio and the promotion of CytC release from the mitochondria into the cytoplasm. In another study, Liu et al (40) demonstrated that emodin induces Panc-1 cell apoptosis mediated by a decrease in the mitochondrial membrane potential. In addition, emodin at the dose of $40 \mathrm{mg} / \mathrm{kg}$ improved the living state of model mice. In another study, Liu et al (41) found that emodin induced growth inhibition and apoptosis in the pancreatic cancer cell line SW1990 and suppressed the migration and invasion of SW1990 cells in a dose-dependent manner. Emodin significantly downregulated NF- $\kappa$ B DNA-binding activity, survivin and MMP-9 in SW1990 cells. They found that oral administration of emodin significantly decreased tumor weight in vivo and metastasis compared to a control. Furthermore, the expression of $\mathrm{NF}-\kappa \mathrm{B}$, survivin and MMP-9 was also suppressed in tumor tissues treated with emodin. Their results indicated that emodin exerts antiproliferative and antimetastatic activity on pancreatic cancer both in vitro and in vivo, which may be related to downregulation of $\mathrm{NF}-\kappa \mathrm{B}$ and its regulated molecules such as survivin and MMP-9 proteins. Our previous study (42) demonstrated that treatment of gemcitabine combined with emodin efficiently suppressed tumor growth in mice inoculated with pancreatic tumor cells. This treatment paradigm promoted apoptotic cell death and mitochondrial fragmentation. Furthermore, it reduced the phosphorylated-Akt (p-Akt) level, $\mathrm{NF}-\kappa \mathrm{B}$ activation, $\mathrm{Bcl}-2 / \mathrm{Bax}$ ratio, and increased caspase-9/-3 activation and cytochrome $c(\mathrm{CytC})$ release that occurred in combination therapy or by emodin alone. Collectively, emodin enhanced the activity of gemcitabine in tumor growth suppression via inhibition of Akt and NF- $\kappa \mathrm{B}$ activation, thus, promoting the mitochondrial-dependent apoptotic pathway. In addition, Wang et al (26) reported that emodin enhanced the antitumor effect of gemcitabine in SW1990 pancreatic cancer 


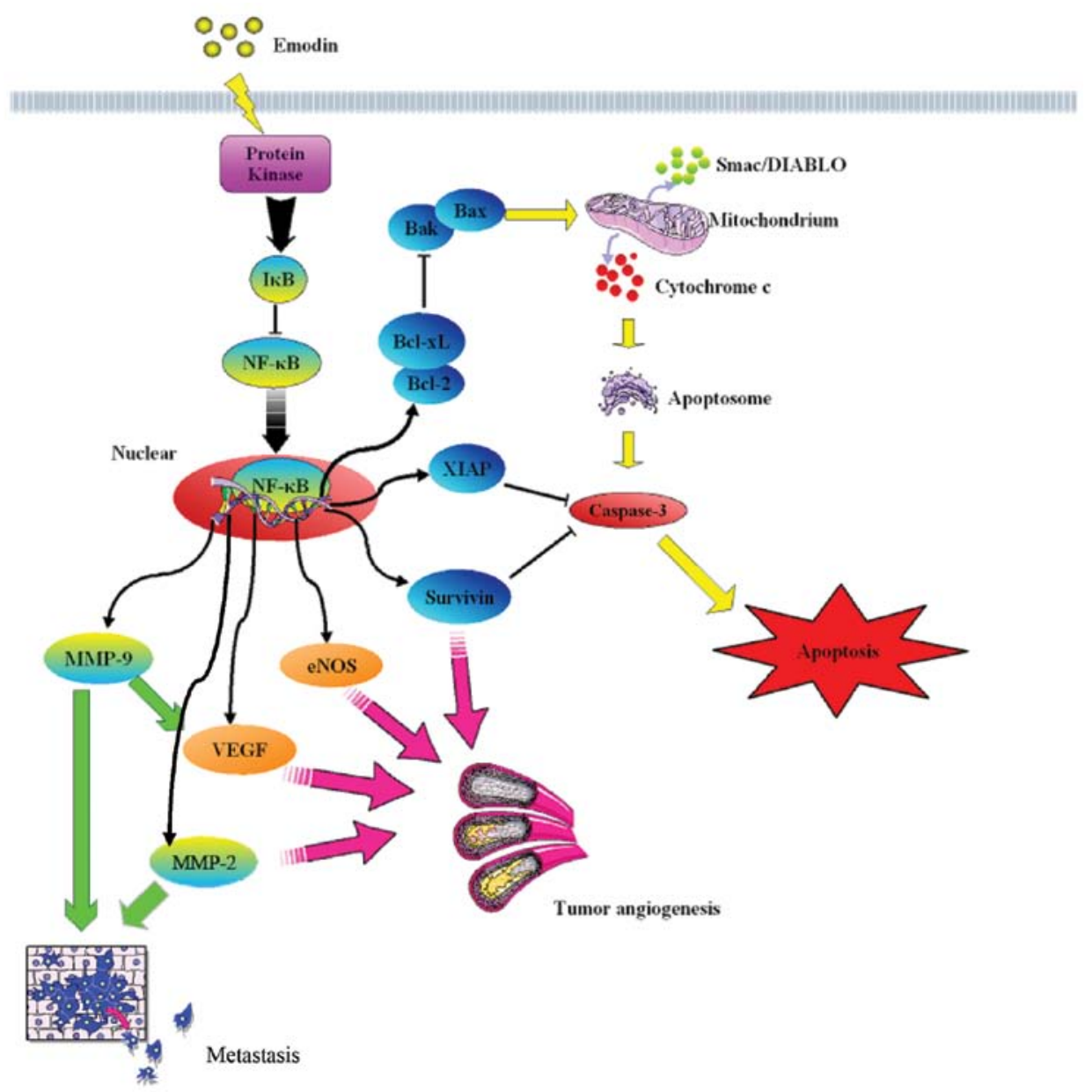

Figure 2. Schematic illustration of possible antitumor mechanisms of emodin against pancreatic cancer.

in vitro and in vivo, which might be associated with downregulation of $\mathrm{NF}-\kappa \mathrm{B}$ expression, thus inhibiting the expression of XIAP. Liu et al (43) reported that emodin promoted cell apoptosis of the gemcitabine-resistant cell line SW1990/Gem in a dose-dependent manner and enhanced the SW1990/Gem cell sensitivity to gemcitabine in a time-dependent manner. They found that emodin monotherapy or combination with gemcitabine decreased the gene and protein expression levels of multi-drug resistance 1 (MDR-1), NF- $\kappa \mathrm{B}$ and $\mathrm{Bcl}-2$ and inhibited the function of $\mathrm{P}-\mathrm{gp}$, but increased the expression levels of Bax, cytochrome $c$ (cytosol), caspase-9 and -3, thus promoting cell apoptosis. This study demonstrated that emodin had a reversing effect on the gemcitabine-resistant cell line SW1990/Gem, possibly via decrease in the function of P-gp and activation of the mitochondrial apoptosis pathway. As members of apoptosis inhibitors, survivin and XIAP play an important role in chemotherapy resistance in pancreatic cancer. Guo et al (44) reported that emodin potentiates the antitumor effects of gemcitabine in PANC-1 cell xenografts via downregulating expression of survivin and XIAP leading to apoptosis. Recently, our study demonstrated that emodin inhibited tumor angiogenesis in vitro and in implanted pancreatic cancer tissues, decreased the expression of angiogenesis-associated factors $(\mathrm{NF}-\kappa \mathrm{B}$ and its regulatory factors VEGF, MMP-2, MMP-9 and eNOS) and reduced eNOS phos- phorylation. In addition, we found that emodin had no effect on VEGFR expression in vivo (13). In summary, these results suggest that emodin has potential antitumor effect on pancreatic cancer via its dual role in the promotion of apoptosis and suppression of angiogenesis, probably through regulation of the expression of NF- $\kappa \mathrm{B}, \mathrm{NF}-\kappa \mathrm{B}$-regulated apoptosis-associated and angiogenesis-associated factors. However, more intense research at the animal level and clinical trials are required to confirm the clinical antitumor efficacy.

\section{Effects of emodin on respiratory system cancer and the possible mechanisms involved}

The recombinant protein $\operatorname{Rad} 51$ is essential for homologous recombination repair, and $\operatorname{Rad} 51$ overexpression is resistant to DNA double-strand break-inducing cancer therapies. Chen et al (45) reported that emodin enhanced the cytotoxicity induced by gefitinib in two non-small cancer lung cancer (NSCLC) cell lines, A549 and H1650. Emodin at low doses of 2-10 $\mu \mathrm{M}$ enhanced a gefitinib-induced decrease in phosphoERK1/2 and Rad51 protein levels by enhancing Rad51 protein instability. Expression of constitutively active MKK1/2 vectors (MKK1/2-CA) significantly rescued the reduced phosphoERK1/2 and Rad51 protein levels as well as cell viability following gefitinib and emodin cotreatment. Blockage of 
ERK1/2 activation by U0126 (an MKK1/2 inhibitor) lowered Rad51 protein levels and reduced cell viability in emodintreated H1650 and A549 cells. ROS have been implicated in the phosphorylation of $\mathrm{p} 53$ that is mediated by protein kinases, including p38MAPK, ataxia-telangiectasia mutated (ATM) and ERK (46). Lai et al (47) demonstrated that emodin induced mitochondria-dependent apoptotic cell death in human lung adenocarcinoma A549 cells by activating a ROS-activated ATM-p53-Bax signaling pathway. ERCC1 and Rad51 proteins are essential for nucleotide excision repair and homologous recombination, respectively. Furthermore, ERCC1 and Rad51 overexpression induces resistance to DNA-damaging agents that promote DNA double-strand breaks. Both ERCC1 and Rad51 protein levels as well as mRNA levels were decreased in 4 different NSCLC cell lines after exposure to emodin. These decreased levels were correlated with the inactivation of the MKK1/2-ERK1/2 pathway (48). The most important chemotherapeutic agents for patients with advanced NSCLC are platinum-containing compounds such as cisplatin or carboplatin. Resistance to platinum-based drugs is one of the major obstacles to cancer treatment, and is often related to poor prognosis in patients with NSCLC. Emodin was found to synergistically increase the cytotoxicity induced by cisplatin in human NSCLC cell lines (49). Exposure of human NSCLC cells to emodin decreased cisplatin-elicited ERK1/2 activation and ERCC1 protein induction by increasing instability of ERCC1 protein. Su et al (50) reported that exposure of the human NSCLC H1703 or A549 cell lines to emodin decreased the antitumor antibiotic mitomycin C (MMC)elicited phosphorylated ERK1/2 and Rad51 levels. Moreover, emodin significantly decreased the MMC-elicited Rad51 mRNA and protein levels by increasing the instability of Rad51 mRNA and protein. Thymidine phosphorylase (TP) is the rate-limiting enzyme for the activation of capecitabine (a pro-drug of fluorouracil), and is a useful predictor of tumor response to capecitabine-based chemotherapy. Overexpression of Rad51 and ERCC1 induces resistance to chemotherapeutic agents. Ko et al (51) reported that emodin enhanced capecitabine-induced cytotoxic effects through ERK1/2 inactivation and decreased the Rad51 and ERCC1 protein levels induced by capecitabine. Enhancement of ERK1/2 signaling by constitutively active MKK1/2 (MKK1/2-CA) resulted in increasing Rad51 and ERCC1 protein levels and cell viability in NSCLC cell lines treated with emodin and capecitabine. Interestingly, emodin enhanced TP mRNA and protein expression in capecitabine-treated NSCLC cell lines, and depletion of the TP expression decreased the cytotoxic effects induced by capecitabine and emodin. These findings suggest that the enhanced cytotoxicity to capecitabine by emodin was mediated by downregulation of the expression of $\operatorname{Rad} 51$ and ERCC1 and upregulation of TP expression. He et al (52) found that emodin exerted a suppressive effect on the proliferation of NSCLC in a concentration-dependent manner. Protein and mRNA expression of ERCC1 and Rad51 was also significantly decreased with the dose. Vacuolar degeneration was observed in A549 and SK-MES-1 cell lines after emodin treatment by transmission electron microscopy. The authors concluded that emodin may thus inhibit the cell proliferation of NSCLC cells by downregulation of RCC1 and Rad51.

\section{Effects of emodin on reproductive system cancer and the possible mechanisms involved}

Breast cancer. Cheema et al (53) reported that the drug delivery and efficacy of silk fibroin-coated liposomes (SF-ELP) encapsulating the receptor tyrosine kinase inhibitor, emodin, inhibited both the PI3K and MAPK pathways and reduced levels of phosphorylated Her2/neu, which contribute to the enhanced growth inhibitory effects of Her2/neu-overexpressing breast cancer cells. The present study suggested that silk fibroin coating enhanced emodin delivery in Her2/ neu-overexpressing breast cancer cells thereby increasing the overall efficacy of the drug. Huang et al (54) demonstrated that emodin induced apoptosis through a decrease in the Bcl-2/Bax ratio and an increase in cytoplasm cytochrome $c$ concentration in human breast cancer BCap-37 cells. Furthermore, Huang et al (55) demonstrated that emodin induced alterations in gene expression profiling, but had no effects on caspases. In addition, the p53 pathway may cooperate with the IGF-2 pathway, resulting in emodin-induced apoptosis through disruption of the mitochondrial signaling pathway in BCap-37 cells. Wang et al (56) prepared emodin-loaded solid lipid nanoparticles (E-SLNs) by high pressure homogenization $(\mathrm{HPH})$ and evaluated their antitumor activity in vitro against human breast cancer cell lines. The drug release of E-SLNs in vitro lasted $72 \mathrm{~h}$ and exhibited a sustained profile, which makes it a promising vehicle for oral drug delivery. Their study showed that E-SLNs significantly enhanced the in vitro cytotoxicity against human breast cancer cell lines MCF-7 and MDA-MB-231 when compared to the emodin solution, while free emodin, blank SLNs (B-SLNs) and E-SLNs all exhibited no significant toxicity to human mammary epithelial MCF-10A cells. E-SLNs also showed a highly significant cell cycle arrest effect in MCF-7 cells compared to bulk emodin solution and induced higher apoptotic rates in MCF-7 cells, indicating that cell cycle arrest and apoptosis may be the underlying mechanism of the enhanced cytotoxicity. These results suggest that the delivery of emodin by lipid nanoparticles may be a promising approach for cancer therapy.

Cervical cancer. Treatment of eukaryotic cells with emodin, which is described as an inhibitor of protein kinase CK2, was found to induce apoptosis and the anti-apoptotic effect of CK2 is partially mediated by targeting phosphorylation and upregulation of AKT by CK2. Oslen et al (57) further showed that the downregulation of AKT in the HeLa cell line was partially due to the emodin-mediated target inhibition of components of the PI3K pathway, which directly or indirectly affect AKT activity. More studies are needed to demonstrate the effect of emodin in cervical cancer.

Prostate cancer. Huang et al (58) found that emodin/cisplatin co-treatment markedly elevated ROS levels and enhanced chemosensitivity in DU-145 cells, when compared with cisplatin-only treatment, but exerted little effect on non-tumor cells. The mechanism may be associated with downregulation of MDR1 expression and promotion of drug retention and suppression of transactivation of HIF-1. Their results suggest that emodin may be recognized an an effective adjunctive 
to improve the efficacy of cytotoxic drugs in prostate cancer cells with over-activated HIF-1 and high MDR. Yu et al (59) reported that emodin caused a marked increase in apoptosis and a decrease in cell proliferation in human prostate cancer $\mathrm{LNCaP}$ cells. The expression of the androgen receptor (AR) and prostate-specific antigen (PSA) was decreased and the expression of p53 and p21 was increased as the emodin concentrations were increased. At the same time, emodin induced apoptosis of $\mathrm{LNCaP}$ cells through the upregulation of caspase- 3 and -9 , as well as the increase in the Bax/Bcl-2 ratio. However, it did not involve modulation of Fas or caspase-8 protein expression. This study concluded that emodin inhibits cell proliferation by AR and p53-p21 pathways and induces apoptosis via the mitochondrial pathway. Recent evidence indicates that the CXCR4/CXCL12 axis is involved in promoting invasion and metastasis in tumors. Thus, novel agents that downregulate CXCR4 expression have therapeutic potential for suppressing cancer metastasis. Ok et al (60) reported that emodin downregulated the expression of both CXCR4 and HER2 without affecting the cell viability of tumor cells. The suppression of CXCR4 expression by emodin was found to correlate with the inhibition of CXCL12-induced migration and invasion of both prostate and lung cancer DU145 and A549 cells. Their findings suggest that emodin is a novel blocker of CXCR4 expression and, thus, has enormous potential as a powerful therapeutic agent for metastatic cancer.

Ovarian cancer. The onset of drug resistance is a major impediment toward the successful treatment of ovarian cancer. Li et al (61) showed that emodin alone induced apoptosis at a high concentration and increased paclitaxelinduced apoptosis at a low concentration. It enhanced the sensitivity of A2780/taxol cells to paclitaxel along with downregulation of P-glycoprotein, XIAP and survivin. Their results demonstrated a dual role for emodin in the inhibition of drug-resistant ovarian tumor growth by increasing paclitaxel cellular concentrations and re-sensitizing resistant cells to paclitaxel. Their results suggest the possibility of an innovative chemotherapeutic strategy that uses emodin in combination with paclitaxel to increase the sensitivity of tumor cells. Human ovarian A2780 cells were exposed first to emodin or $\mathrm{DRB}$ and then to cisplatin alone, and the cytotoxic effects were monitored. The cellular effects of cisplatin were significantly enhanced, whereas simultaneous exposure did not enhance the cytotoxicity. The increase in activity of cisplatin in the sequenced schedule was not due to increases in intracellular levels of cisplatin or DNA adducts, whereas the cytotoxic inhibition was related to a significant decline in both intracellular platinum levels and DNA adducts, which were ascribed to inhibition of cisplatin uptake. Emodin in combination with cisplatin inhibits platinum drug uptake by impacting the hCtrl transporter thereby reducing the cytotoxicity of cisplatin (62).

\section{Effects of emodin on blood system cancer and the possible mechanisms involved}

Muto et al (7) demonstrated that emodin significantly induced cytotoxicity in human myeloma cells through the elimination of myeloid cell leukemia 1 (Mcl-1). Emodin inhibited interleukin-6-induced activation of Janus-activated kinase 2 (JAK2) and phosphorylation of signal transducer and activator of transcription 3 (STAT3), followed by the decreased expression of Mcl-1. Activation of caspase-3 and caspase- 9 was triggered by emodin, while the expression of other antiapoptotic Bcl-2 family members, except Mcl-1, were not altered in the presence of emodin. Induction of apoptosis by emodin was almost abrogated in Mcl-1-overexpressing myeloma cells similar to the level in parental cells, which were not treated with emodin, suggesting the importance of Mcl-1 in emodin-induced apoptosis. The use of arsenic trioxide $\left(\mathrm{As}_{2} \mathrm{O}_{3}\right)$ has been shown to effectively treat acute promyelocytic leukemia (APL) with $>80 \%$ of patients achieving complete remission. The combination of arsenic and interferon has also shown promising results in the treatment of adult T-cell leukemia (ATL). The requirement for slow dose increases of arsenic and the time required to achieve a pharmacologic active dose in patients are major obstacles as the median survival of patients with ATL is approximately 6 months. Brown et al (63) reported a potent synergistic effect of the combination of arsenic trioxide and interferon $\alpha(\mathrm{As} / \mathrm{IFN}-\alpha)$ with emodin on cell-cycle arrest and cell death of HTLV-I-infected cells via the generation of ROS and inhibition of Akt and AP-1. Importantly, clinically achievable doses of emodin allowed for reduced arsenic concentrations by 100 -fold while still achieving a high toxic effect on tumor cells. Wang et al (64) reported that emodin treatment may result in apoptosis of human chronic myelocytic leukemia K562 cells. Both in vitro and in vivo, the apoptosisrelated protein $\mathrm{Bcl}-2$ was decreased and the Bax was increased after emodin treatment. Moreover, activation of caspase- $3,-8$ and -9 by emodin has been demonstrated.

\section{Conclusions}

Overall, based on analyses of data from in vitro or in vivo laboratory experimental models, we suggest that emodin is a potent anticancer agent for cancer treatment particularly in digestive system cancers such as pancreatic cancer by regulating multi-molecular targets involved in tumor growth, invasion, angiogenesis and metastasis. Studies in recent years indicate that emodin has an anticancer effect in several types of cancer, yet the mechanism of action in each type of cancer appears to vary. Promoting apoptosis through the mitochondrial pathway may be a similar antitumor mechanism of emodin in different types of cancer from different tissues. This review provides information regarding the promising anticancer actions of emodin in cancer treatment and aimed to broaden the therapeutic potential to cancer patients in the future. However, despite the encouraging results summarized here, further studies concerning the antitumor mechanisms of emodin and clinical trials are warranted and required to confirm the clinical antitumor efficacy before the possibility of large-scale clinical application.

\section{Acknowledgements}

We are grateful for the funding support from the Administration of Traditional Chinese Medicine of Zhengjing Province, China (grant no. 2011ZZ010), the Zhejiang Provincial Science Fund for Distinguished Young Scholars (grant no. LR12H280001) and the National Natural Science Foundation of China (grant no. 81173606). 


\section{References}

1. Chen YC, Shen SC, Lee WR, et al: Emodin induces apoptosis in human promyeloleukemic HL-60 cells accompanied by activation of caspase 3 cascade but independent of reactive oxygen species production. Biochem Pharmacol 64: 1713-1724, 2002.

2. Su YT, Chang HL, Shyue SK and Hsu SL: Emodin induces apoptosis in human lung adenocarcinoma cells through a reactive oxygen species-dependent mitochondrial signaling pathway. Biochem Pharmacol 70: 229-241, 2005.

3. Srinivas G, Anto RJ, Srinivas P, Vidhyalakshmi S, Senan VP and Karunagaran D: Emodin induces apoptosis of human cervical cancer cells through poly(ADP-ribose) polymerase cleavage and activation of caspase-9. Eur J Pharmacol 473: 117-125, 2003.

4. Huang Q, Shen HM, Shui G, Wenk MR and Ong CN: Emodin inhibits tumor cell adhesion through disruption of the membrane lipid Raft-associated integrin signaling pathway. Cancer Res 66 5807-5815, 2006.

5. Kwak HJ, Park MJ, Park CM, et al: Emodin inhibits vascular endothelial growth factor-A-induced angiogenesis by blocking receptor-2 (KDR/Flk-1) phosphorylation. Int J Cancer 118: 2711-2720, 2006

6. Cha TL, Qiu L, Chen CT, Wen Y and Hung MC: Emodin downregulates androgen receptor and inhibits prostate cancer cell growth. Cancer Res 65: 2287-2295, 2005.

7. Muto A, Hori M, Sasaki Y, et al: Emodin has a cytotoxic activity against human multiple myeloma as a Janus-activated kinase 2 inhibitor. Mol Cancer Ther 6: 987-994, 2007.

8. Kim MS, Park MJ, Kim SJ, et al: Emodin suppresses hyaluronic acid-induced MMP-9 secretion and invasion of glioma cells. Int J Oncol 27: 839-846, 2005.

9. Bader Y, Hartmann J, Horvath Z, et al: Synergistic effects of deuterium oxide and gemcitabine in human pancreatic cancer cell lines. Cancer Lett 259: 231-239, 2008.

10. Nakagawa T, Shimizu M, Shirakami Y, et al: Synergistic effects of acyclic retinoid and gemcitabine on growth inhibition in pancreatic cancer cells. Cancer Lett 273: 250-256, 2009.

11. Lee SH, Ryu JK, Lee KY, et al: Enhanced anti-tumor effect of combination therapy with gemcitabine and apigenin in pancreatic cancer. Cancer Lett 259: 39-49, 2008.

12. Wang SJ, Gao Y, Chen H, et al: Dihydroartemisinin inactivates $\mathrm{NF}-\mathrm{\kappa B}$ and potentiates the anti-tumor effect of gemcitabine on pancreatic cancer both in vitro and in vivo. Cancer Lett 293: 99-108, 2010.

13. Lin SZ, Wei WT, Chen H, et al: Antitumor activity of emodin against pancreatic cancer depends on its dual role: promotion of apoptosis and suppression of angiogenesis. PLoS One 7: e42146, 2012.

14. Lin SY, Lai WW, Ho CC, et al: Emodin induces apoptosis of human tongue squamous cancer SCC-4 cells through reactive oxygen species and mitochondria-dependent pathways. Anticancer Res 29: 327-335, 2009.

15. Chen YY, Chiang SY, Lin JG, et al: Emodin, aloe-emodin and rhein induced DNA damage and inhibited DNA repair gene expression in SCC-4 human tongue cancer cells. Anticancer Res 30: 945-951, 2010

16. Chen YY, Chiang SY, Lin JG, et al: Emodin, aloe-emodin and rhein inhibit migration and invasion in human tongue cancer SCC- 4 cells through the inhibition of gene expression of matrix metalloproteinase-9. Int J Oncol 36: 1113-1120, 2010.

17. Cai J, Niu X, Chen Y, et al: Emodin-induced generation of reactive oxygen species inhibits $R$ hoA activation to sensitize gastric carcinoma cells to anoikis. Neoplasia 10: 41-51, 2008

18. Sun ZH and Bu P: Downregulation of phosphatase of regenerating liver-3 is involved in the inhibition of proliferation and apoptosis induced by emodin in the SGC-7901 human gastric carcinoma cell line. Exp Ther Med 3: 1077-1081, 2012.

19. Wang QJ, Cai XB, Liu MH, Hu H, Tan XJ and Jing XB: Apoptosis induced by emodin is associated with alterations of intracellular acidification and reactive oxygen species in EC-109 cells. Biochem Cell Biol 88: 767-774, 2010.

20. Hsu CM, Hsu YA, Tsai Y, et al: Emodin inhibits the growth of hepatoma cells: finding the common anti-cancer pathway using Huh7, Hep3B, and HepG2 cells. Biochem Biophys Res Commun 392: 473-478, 2010.

21. Yu JQ, Bao W and Lei JC: Emodin regulates apoptotic pathway in human liver cancer cells. Phytother Res 27: 251-257, 2012

22. Kim MJ, Oh DY, Lee SH, et al: Gemcitabine-based versus fluoropyrimidine-based chemotherapy with or without platinum in unresectable biliary tract cancer: a retrospective study. BMC Cancer 8: 374, 2008.
23. Wang W, Sun YP, Huang XZ, et al: Emodin enhances sensitivity of gallbladder cancer cells to platinum drugs via glutathion depletion and MRP1 downregulation. Biochem Pharmacol 79: $1134-1140,2010$

24. Trachootham D, Alexandre J and Huang P: Targeting cancer cells by ROS-mediated mechanisms: a radical therapeutic approach? Nat Rev Drug Discov 8: 579-591, 2009.

25. Wang $\mathrm{J}$ and Yi J: Cancer cell killing via ROS: to increase or decrease, that is the question. Cancer Biol Ther 7: 1875-1884, 2008.

26. Wang W, Sun Y, Li X, et al: Emodin potentiates the anticancer effect of cisplatin on gallbladder cancer cells through the generation of reactive oxygen species and the inhibition of survivin expression. Oncol Rep 26: 1143-1148, 2011.

27. Moserle L, Ghisi M, Amadori A and Indraccolo S: Side population and cancer stem cells: therapeutic implications. Cancer Lett 288: 1-9, 2010.

28. $\mathrm{Wu} \mathrm{C}$ and Alman BA: Side population cells in human cancers. Cancer Lett 268: 1-9, 2008.

29. Tabor MH, Clay MR, Owen JH, et al: Head and neck cancer stem cells: the side population. Laryngoscope 121: 527-533, 2011.

30. Li XX, Wang J, Wang HL, et al: Characterization of cancer stem-like cells derived from a side population of a human gallbladder carcinoma cell line, SGC-996. Biochem Biophys Res Commun 419: 728-734, 2012.

31. Li XX, Ding Y, Wang W, et al: Emodin as an effective agent in targeting cancer stem-like side population cells of gallbladder carcinoma. Stem Cells Dev 22: 554-566, 2012.

32. Lu Y,Zhang J and Qian J: The effect of emodin on VEGF receptors in human colon cancer cells. Cancer Biother Radiopharm 23: 222-228, 2008.

33. Ma YS, Weng SW, Lin MW, et al: Antitumor effects of emodin on LS1034 human colon cancer cells in vitro and in vivo: roles of apoptotic cell death and LS1034 tumor xenografts model. Food Chem Toxicol 50: 1271-1278, 2012.

34. Saha S, Bardelli A, Buckhaults P, et al: A phosphatase associated with metastasis of colorectal cancer. Science 294: 1343-1346, 2001.

35. Ooki A, Yamashita K, Kikuchi S, Sakuramoto S, Katada N and Watanabe M: Phosphatase of regenerating liver-3 as a convergent therapeutic target for lymph node metastasis in esophageal squamous cell carcinoma. Int J Cancer 127: 543-554, 2010.

36. Han YM, Lee SK, Jeong DG, et al: Emodin inhibits migration and invasion of DLD-1 (PRL-3) cells via inhibition of PRL-3 phosphatase activity. Bioorg Med Chem Lett 22: 323-326, 2012.

37. Damodharan U, Ganesan R and Radhakrishnan UC: Expression of MMP2 and MMP9 (gelatinases A and B) in human colon cancer cells. Appl Biochem Biotechnol 165: 1245-1252, 2011.

38. Guo Q, Chen Y, Zhang B, Kang M, Xie Q and Wu Y: Potentiation of the effect of gemcitabine by emodin in pancreatic cancer is associated with survivin inhibition. Biochem Pharmacol 77: 1674-1683, 2009.

39. Chen H, Wei W, Guo Y, et al: Enhanced effect of gemcitabine by emodin against pancreatic cancer in vivo via cytochrome C-regulated apoptosis. Oncol Rep 25: 1253-1261, 2011.

40. Liu JX, Zhang JH, Li HH, et al: Emodin induces Panc-1 cell apoptosis via declining the mitochondrial membrane potential. Oncol Rep 28: 1991-1996, 2012.

41. Liu A, Chen H, Wei W, et al: Antiproliferative and antimetastatic effects of emodin on human pancreatic cancer. Oncol Rep 26: 81-89, 2011

42. Wei WI, Chen H, Ni ZL, et al: Antitumor and apoptosispromoting properties of emodin, an anthraquinone derivative from Rheum officinale Baill, against pancreatic cancer in mice via inhibition of Akt activation. Int J Oncol 39: 1381-1390, 2011.

43. Liu DL, Bu H, Li H, et al: Emodin reverses gemcitabine resistance in pancreatic cancer cells via the mitochondrial apoptosis pathway in vitro. Int J Oncol 40: 1049-1057, 2012.

44. Guo HC, Bu HQ, Luo J, et al: Emodin potentiates the antitumor effects of gemcitabine in PANC-1 pancreatic cancer xenograft model in vivo via inhibition of inhibitors of apoptosis. Int J Oncol 40: 1849-1857, 2012.

45. Chen RS, Jhan JY, Su YJ, et al: Emodin enhances gefitinibinduced cytotoxicity via Rad51 downregulation and ERK1/2 inactivation. Exp Cell Res 315: 2658-2672, 2009.

46. Liu B, Chen Y and St Clair DK: ROS and p53: a versatile partnership. Free Radic Biol Med 44: 1529-1535, 2008.

47. Lai JM, Chang JT, Wen CL and Hsu SL: Emodin induces a reactive oxygen species-dependent and ATM-p53-Bax mediated cytotoxicity in lung cancer cells. Eur J Pharmacol 623: 1-9, 2009. 
48. Ko JC, Su YJ, Lin ST, et al: Suppression of ERCC1 and Rad51 expression through ERK1/2 inactivation is essential in emodinmediated cytotoxicity in human non-small cell lung cancer cells. Biochem Pharmacol 79: 655-664, 2010.

49. Ko JC, Su YJ, Lin ST, et al: Emodin enhances cisplatin-induced cytotoxicity via down-regulation of ERCC1 and inactivation of ERK1/2. Lung Cancer 69: 155-164, 2010.

50. Su YJ, Tsai MS, Kuo YH, et al: Role of Rad51 down-regulation and extracellular signal-regulated kinases 1 and 2 inactivation in emodin and mitomycin C-induced synergistic cytotoxicity in human non-small-cell lung cancer cells. Mol Pharmacol 77: 633-643, 2010

51. Ko JC, Tsai MS, Kuo YH, et al: Modulation of Rad51, ERCC1, and thymidine phosphorylase by emodin result in synergistic cytotoxic effect in combination with capecitabine. Biochem Pharmacol 81: 680-690, 2011.

52. He L, Bi JJ, Guo Q, Yu Y and Ye XF: Effects of emodin extracted from Chinese herbs on proliferation of non-small cell lung cancer and underlying mechanisms. Asian Pac J Cancer Prev 13: $1505-1510,2012$.

53. Cheema SK, Gobin AS, Rhea R, Lopez-Berestein G,Newman RA and Mathur AB: Silk fibroin mediated delivery of liposomal emodin to breast cancer cells. Int J Pharm 341: 221-229, 2007.

54. Huang Z, Chen G and Shi P: Emodin-induced apoptosis in human breast cancer BCap-37 cells through the mitochondrial signaling pathway. Arch Pharm Res 31: 742-748, 2008.

55. Huang Z, Chen G and Shi P: Effects of emodin on the gene expression profiling of human breast carcinoma cells. Cancer Detect Prev 32: 286-291, 2009.

56. Wang S, Chen T, Chen R, Hu Y, Chen M and Wang Y: Emodin loaded solid lipid nanoparticles: preparation, characterization and antitumor activity studies. Int J Pharm 430: 238-246, 2012.
57. Olsen BB, Bjorling-Poulsen M and Guerra B: Emodin negatively affects the phosphoinositide 3-kinase/AKT signalling pathway: a study on its mechanism of action. Int J Biochem Cell Biol 39: 227-237, 2007

58. Huang XZ, Wang J, Huang C, et al: Emodin enhances cytotoxicity of chemotherapeutic drugs in prostate cancer cells: the mechanisms involve ROS-mediated suppression of multidrug resistance and hypoxia inducible factor-1. Cancer Biol Ther 7: 468-475, 2008.

59. Yu CX, Zhang XQ, Kang LD, et al: Emodin induces apoptosis in human prostate cancer cell LNCaP. Asian J Androl 10: 625-634, 2008.

60. Ok S, Kim SM, Kim C, et al: Emodin inhibits invasion and migration of prostate and lung cancer cells by downregulating the expression of chemokine receptor CXCR4. Immunopharmacol Immunotoxicol 4: 768-778, 2012.

61. Li J, Liu P, Mao H, Wanga A and Zhang X: Emodin sensitizes paclitaxel-resistant human ovarian cancer cells to paclitaxelinduced apoptosis in vitro. Oncol Rep 21: 1605-1610, 2009.

62. Kurokawa T, He G and Siddik ZH: Protein kinase inhibitors emodin and dichloro-ribofuranosylbenzimidazole modulate the cellular accumulation and cytotoxicity of cisplatin in a scheduledependent manner. Cancer Chemother Pharmacol 65: 427-436, 2010.

63. Brown M, Bellon M and Nicot C: Emodin and DHA potently increase arsenic trioxide interferon- $\alpha$-induced cell death of HTLV-I-transformed cells by generation of reactive oxygen species and inhibition of Akt and AP-1. Blood 109: 1653-1659, 2007.

64. Wang C-G, Yang J-Q, Liu B-Z, et al: Anti-tumor activity of emodin against human chronic myelocytic leukemia K562 cell lines in vitro and in vivo. Eur J Pharmacol 627: 33-41, 2010. 